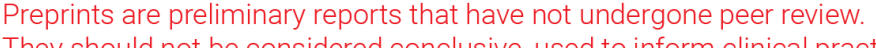 They should not be considered conclusive, used to inform clinical practice, or referenced by the media as validated information. \\ Oral Health Related Quality of Life during Pregnancy and Postpartum: A systematic review
}

\section{Omid Fakheran}

Isfahan University of Medical Sciences

\section{Zahra Saied-Moallemi}

Isfahan University of Medical Sciences

Abbasali Khademi ( $\Delta$ omid_goodhero@yahoo.com )

isfahan university of medical sciences https://orcid.org/0000-0003-0994-0288

\section{Research article}

Keywords: Pregnancy, Postpartum Period, Oral Health, Health Related Quality Of Life, Dental Caries, Periodontal Diseases, Gingival Diseases, Odontalgia, Pain

Posted Date: July 17th, 2019

DOI: https://doi.org/10.21203/rs.2.11594/v1

License: (c) (1) This work is licensed under a Creative Commons Attribution 4.0 International License. Read Full License 


\section{Abstract}

BACKGROUND The impact of diseases on psychosocial well-being or the normal function of a person has been commonly defined as health-related quality of life. High prevalence of Dental and periodontal problems during gestation period may have a negative effect on oral-health-related quality of life (OHRQoL) in pregnant and postpartum women. OBJECTIVE This systematic review aims to perform a quality assessment and to give a critical overview of the current research available on OHRQoL in pregnant and post-partum women. STUDY DESIGN A systematic search strategy was applied in PubMed, MEDLINE, Cochrane Library, Web of Science, ClinicalTrials.gov, Google Scholar and Scopus from inception until December 2018. For this systemic review all original and peer-reviewed human studies which investigated OHRQoL of women during pregnancy or post- partum period were searched. Studies were screened on title and consecutively on abstract for relevance by 2 independent investigators. Methodological quality was assessed using modified items recommended by the Newcastle-Ottawa Scale for observational studies. RESULTS In all, 8 studies regarding the assessment of OHRQoL in pregnant woman were included. All of the included studies had cross-sectional design. Meta-analysis was not possible due to heterogeneity on key aspects among the included studies. Thus, the data from the studies were evaluated qualitatively. The overall risk of bias of the included studies was low. CONCLUSION The main conclusion of this review is that the presence of signs and symptoms of dental and gingival disease negatively affects the self-perception of OHRQoL in pregnant women. The most affected domains of OHRQoL in pregnant women were related to mental and psychological discomfort followed by physical and functional problems. Considering that the available evidence is limited to crosssectional design, longitudinal studies are needed to investigate the impacts of oral health status during pregnancy.

\section{Abstract}

\section{BACKGROUND:}

The impact of diseases on psychosocial well-being or the normal function of a person has been commonly defined as health-related quality of life .High prevalence of Dental and periodontal problems during gestation period may have a negative effect on oral-health-related quality of life (OHRQoL) in pregnant and postpartum women.

\section{OBJECTIVE:}

This systematic review aims to perform a quality assessment and to give a critical overview of the current research available on OHRQoL in pregnant and post-partum women.

\section{STUDY DESIGN:}

A systematic search strategy was applied in PubMed, MEDLINE, Cochrane Library, Web of Science, ClinicalTrials.gov, Google Scholar and Scopus from inception until December 2018. For this systemic 
review all original and peer-reviewed human studies which investigated OHRQoL of women during pregnancy or post- partum period were searched. Studies were screened on title and consecutively on abstract for relevance by 2 independent investigators. Methodological quality was assessed using modified items recommended by the Newcastle-Ottawa Scale for observational studies.

\section{RESULTS:}

In all, 8 studies regarding the assessment of OHRQoL in pregnant woman were included. All of the included studies had cross-sectional design. Meta-analysis was not possible due to heterogeneity on key aspects among the included studies. Thus, the data from the studies were evaluated qualitatively. The overall risk of bias of the included studies was low.

\section{CONCLUSION:}

The main conclusion of this review is that the presence of signs and symptoms of dental and gingival disease negatively affects the self-perception of OHRQoL in pregnant women. The most affected domains of OHRQoL in pregnant women were related to mental and psychological discomfort followed by physical and functional problems. Considering that the available evidence is limited to cross-sectional design, longitudinal studies are needed to investigate the impacts of oral health status during pregnancy.

\section{Key words:}

Pregnancy, Postpartum Period, Oral Health, Health Related Quality Of Life, Dental Caries, Periodontal Diseases, Gingival Diseases, Odontalgia, Pain

\section{Plain English Summary:}

Pregnancy is a period which some changes occur in physical and emotional status of women's health. These alterations lead to notable changes in the oral environment and cause some painful clinical signs and symptoms.

Feeling pain in oral cavity, bleeding and redness of gums and also changes in sensation of taste are the common problems reported by pregnant women. It is conceivable that these symptoms may impair the perceptions of oral condition and Oral Health Related Quality of Life (OHRQoL). Despite the existence of many studies regarding the objective assessment of oral health status in pregnant women, there is no consensus report regarding the status of OHRQoL in pregnant women. This is the first systematic review concerning OHRQoL in pregnant and post-partum women. It takes into account the quality of studies and the most affected domains of OHRQoL in pregnant women.

Our objective was to perform a quality assessment and to give a critical overview of the current research available on OHRQoL in pregnant and post-partum women.in this paper we also review and explain all the instruments and questionnaires employed for assessing OHRQoL up to now. 
Among many instruments, three of them have been used for measuring OHRQoL in pregnant women up to now. It should be noticed that none of these questionnaires are specifically designed and developed for pregnant women.

The major conclusion of this review is that the presence of signs and symptoms of dental and gingival disease negatively affects the self-perception of OHRQoL in pregnant women.

\section{Introduction}

During the period of pregnancy the body undergoes some important hormonal and physiological changes which could be associated to some alteration in oral health status(1). Moreover the alteration in physical and emotional demands of a pregnant woman might contribute to the neglect in oral hygiene behaviors and develop some problems( 2,3). Dental caries, pregnancy epulis, Gingivitis and periodontal disease are the most common problems that pregnant women face (4-6).

The main etiological factor of dental caries and periodontal disease is the ecology or the bacterial flora of dental plaque. During pregnancy, the immunological and vascular changes due to alteration in endogenous steroid hormones, amplify the inflammatory response in presence of dental plaque and calculus $(7,8)$. Poor oral hygiene cumulated with systemic changes in the body of pregnant women may lead to high prevalence of dental caries and periodontal disease in gestation period (9-11).

Dental and periodontal problems during pregnancy are not usually fatal, even so feeling pain or discomfort in oral cavity, functional limitations, smiling and social relationships due to teeth appearance or soft tissue problems tend to have a substantial effect on individual wellbeing( 12).

The impact of diseases on psychosocial well-being or the normal function of a person has been commonly defined as health-related quality of life $(13,14)$. High prevalence of oral diseases may have a negative effect on oral-health-related quality of life (OHRQoL) in pregnant and postpartum women. The OHRQoL of women during the gestation period affects maternal health as well as fetal and infant health( 15).

The impact of oral health status of pregnant women on their quality of life has been investigated less than other health issues such as emotional and systemic disease. Identifying the objective effect of oral disease on pregnant women and their perception in this regard can help ensure treatment and care that fits the needs and concerns of the patient.

The aim of present study was to conduct a systematic review of the literature to find more consistent evidence regarding the impact of oral health status of pregnant and post-partum women on their OHRQoL.

\section{Materials And Methods}


This study was prepared in accordance with preferred reporting items for systemic Reviews and metaanalyses (PRISMA) guideline (16)and was registered in PROSPERO (ID: CRD42019122148).

\section{Search strategy and data source}

A comprehensive search of 8 electronic databases was conducted, including: PubMed (http://www.ncbi.nlm.nih.gov/pubmed) , MEDLINE, ClinicalTrials.gov (https://www.clinicaltrials.gov )Web of Science (http://www.isiknowledge.com), Google Scholar ( https://scholar.google.com ), Cochrane central (https://www.cochranelibrary.com ) and Scopus ( https://www.scopus.com ).

These databases were searched using the following strategy and keywords: ("quality of life ") AND ("oral health" OR "Dental Caries" OR "Periodontal Diseases" OR " Gingival Diseases " OR " Odontalgia " OR " Pain ") AND ("Pregnancy" OR "Pregnant" OR “Postpartum”). This search was not restricted by date or language. In addition, the proceedings of several scientific meetings were hand-checked and the reference lists of all selected articles were also reviewed for potentially relevant studies.

\section{Study selection}

Citations found through systematic search were inserted to a Microsoft Excel Software 2010 (Microsoft, Redmond, WA). Titles and abstracts were reviewed by two reviewers (OF and ZSM), independently. After the abstract selection, full-text copies of the selected papers were retrieved and the final selection for inclusion was made. After both reviewers performed the complete selection procedure, the results of the searches were compared and discussed in case of disagreement.

\section{Eligibility criteria}

For this systemic review all original and peer-reviewed human studies which investigated OHRQoL of women during pregnancy or post- partum period were searched. For the selection of studies, predefined criteria were used.

All English-written studies providing quantitative information about the status of OHRQoL in pregnant women assessed by a validated questionnaire were included. All the case reports, Letters to the editors, conference proceedings, unpublished studies, narrative reviews and duplicate articles were excluded. When multiple papers were identified on the same population, the study with more information on the data was included in the present review. Studies using general (health related) quality of life measures were excluded. Our search strategy was not restricted to the publication date. Hence all the related evidence up to December 2018 which met the inclusion criteria was assessed. In Fig.1, the flowchart of the study selection is presented.

Fig. 1.Study selection process: PRISMA flowchart of four phases of review showing number of studies identified, selected, eligible and included.

\section{Data collection:}


From the final set of selected studies, the following data were extracted: study characteristics (first author, country where the study was conducted, publication year, study design number of cases and controls, type of sampling, sampling method (randomized or non-randomized), sample size calculation, mean and range of age, Clinical parameters (Probing depth, attachment loss, DMFT and etc.), status of adjustment for confounding factors and the name of questionnaire used for measuring OHRQoL. Regarding the OHRQOL measure, the mean and standard deviations of OHRQOL as well as the number of subjects per subgroups were extracted. Meta-analysis was not possible. Thus, the data from the studies were evaluated qualitatively.

\section{Methodological quality assessment:}

Methodological quality was assessed using modified items recommended by the Newcastle-Ottawa Scale for observational studies $(17,18)$. As cross-sectional studies were included in this systematic review, a modified version of the cohort and case-control study scales was employed (Appendix 1). The following items were measure for each study: representativeness of the sample (evaluated by the sample generation methods and sample origin, e.g., community, specific population groups); comparability (evaluated by the presence of a control group); outcome (outcome assessment tool; concealment for evaluation of outcome; adjustment for confounders and non-response rate). A percentage score was established for each study in accordance with the number of items present (Appendix 1).

\section{Results}

Tables 1 and 2 describe the methodology and main findings of the studies. All the included studies had cross-sectional design regarding to assess OHRQoL in pregnant woman. The most frequent number of studies were conducted in Brazil $(\mathrm{N}=3)$ followed by India $(\mathrm{N}=2)$, China $(\mathrm{N}=1)$, Argentina $(\mathrm{N}=1)$ and Uganda $(\mathrm{N}=1)$. Based on the inclusion criteria, there was not any paper from European or Oceania countries related to our PICO

Table 1 (in the Supplementary Files). Summary of studies: design ,country, type of sample, sampling method, sample size calculation, age, control group, blinded evaluation of outcome, adjustment for confounding factors, Clinical Parameters and OHRQoL measure $(n=8)$.

Table 2 (in the Supplementary Files).Results (Clinical or self-reported criteria related to Oral Health, OHRQoL assessment tool, Main findings, Domains most affected by Pregnancy and OHRQoL Score) of studies included in systematic review $(\mathrm{n}=8)$

\section{Instruments employed for assessment of OHRQoL in Pregnant Women:}

Many Instruments have been applied for assessment of OHRQoL in general population. Among the 10 questionnaire listed in an overview by Slade( 28) three of them have been used in included studies of our systematic review. 
Based on the results which have been shown in table 1 and Table 2, most of the studies used the oral Health Impact profile (OHIP) questionnaire( 29) in both the original 49-item format ( $\mathrm{N}=2)$ and in shortened 14-items version ( $\mathrm{N}=4)$. Among two remaining studies, one of them used The Oral Impacts on Daily Performances (OIDP) questionnaire $(30,31)$ and the other one employed both of OIDP and OHIP-14 questionnaires. The scores obtained from these instruments have been shown in Table 2.

It should be considered that all of the employed questionnaires were translated and validated version of the original forms based on the context of research. Wandera and colleagues in their study used seven of the original eight items of OIDP questionnaire(24). The reason which was mentioned by the authors was the problem in translation of emotional stability item into the local language and possible misinterpretation by the study group. There wasn't any report regarding the addition or elimination of items in translated instruments in other papers.

\section{Domains of OHRQoL Most affected during pregnancy:}

Among the 8 included studies in this review, 6 of them reported the most affected domains of OHRQoL based on the scores obtained from the employed questionnaire. Two of these studies reported the most affected domains based on the OIDP questionnaire( 24, 26, 27). Domains entitled by "Sleeping and relaxing", "cleaning teeth" and "Eating", were the most frequent sections of OIDP questionnaire in both studies.

In the other four studies, the most affected domains were reported based on the OHIP questionnaires. The domains entitled by "Physical pain" and "Psychological discomfort "were grouped as the most affected sections of questionnaires in all 4 studies. Besides these domains, the other domains most affected in pregnant women were "Functional limitation" (3/4 studies), "Physical disability" (2/4 studies) and "Psychological disability" (2/4 studies).

\section{Association of OHRQoL scores with clinical and self-reported parameters:}

With the aim of analyzing the association of OHRQOL with other evidences of oral cavity problems based on the results of included studies, we sorted the disease parameters in two groups of "self-reported items" and "clinical parameters".

Of the 8 studies included in this review, 4 of them employed self-reported items to gathering data related to oral health status of participants. Based on the association analysis of the papers, all of self-reported items except one (self-reported number of teeth) were associated with OHRQoL scores (Table 2).

On the other hand, 7 of 8 studies, used objective clinical parameters to identify the oral health status of women during pregnancy. Among all 7 studies, just the results of one study which was conducted in Argentina didn't show any significant association between OHRQoL scores and clinical findings( 23). 3 of 7 studies showed that positive clinical signs of periodontitis are significantly associated with negative OHRQoL scores. Furthermore, in 3 studies poor OHRQoL was significantly associated with the presence 
of untreated dental caries. Clinical evidence of tooth loss was statistically associated with worst OHRQoL scores in 2 studies.

\section{Discussion}

Despite the different definitions of oral health measures and various instruments used to assess OHRQoL, the major conclusion of this review is that the presence of signs and symptoms of dental and gingival disease negatively affects the self-perception of OHRQoL in pregnant women.

The review confirmed that both "self-reported items" and "clinical parameters" regarding the oral health conditions are more or less associated with OHRQoL. These finding are in accordance with the previous systematic review in general population regarding the association of oral conditions and quality of life( 32). based on the results of both the current review about in pregnant women and the previous review in general population, the Oral Health Impact Profile 14 (OHIP-14)(29) and the Oral Impact on Daily Performance (OIDP) (33) are the 2 most commonly used instruments for evaluation of OHRQoL. This similarity in measurements used for assessing the outcome in general population and specific conditioned people (Pregnant women) can be considered as one of the reasons of accordance in results. These finding might be different if the researchers used condition-specific instruments in their investigations.

The most affected domains of OHRQoL in pregnant women were related to mental and psychological discomfort followed by physical and functional problems. Compared to results obtained from the general population, these findings are in reverse manner( 32 ). The most affected domain in general population was physical health and the psychosocial domains were less affected( 32 ). This discrepancy can be explained by the alteration in emotional and psychological status of women during pregnancy which may affect their self-perception of oral disease. Moreover, employing the different instruments for measuring HRQoL in different studies could be considered as another reason for this discrepancy.

Among all the included studies in this review, just one of them compared the results of pregnant women with non-pregnant control group. This cross-sectional study was conducted among the women with middle and lower socioeconomic status in a rural area of India(19). Based on the results of this study the pregnancy status negatively impacts on OHRQoL scores in almost all domains. More well-controlled studies with larger sample sizes in different contexts should be performed in future to compare the OHRQoL status in pregnant women with non-pregnant women.

The results of this systematic review should be considered under some limitations. Only Papers published in English were included. Comparisons among the studies were difficult, and meta-analysis was not feasible due to heterogeneity on key aspects among the included studies.

First, the exposures were evaluated according to different subjective and objective indices. For the periodontal condition, for example, More than 6 different parameters were adopted in different studies, and the case definitions of tooth loss and dental pain were also distinct. In this regard, collecting a pooled 
estimate for such different parameters is unlikely to provide useful information from a theoretical and clinical perspective.

In addition, the outcome was evaluated by various OHRQoL questionnaires, which were validated in different ways. Finally, in various studies, the investigations were performed among the women with different socioeconomic status and cultural influences.

It should be considered that our main question was looking for the overall status of OHRQoL among pregnant women; therefore all the included articles were cross-sectional studies. It is obvious that the sampling method of a cross-sectional study cannot be random and it should be considered as a limitation. Moreover, Majority of the studies didn't perform adjusted analyses and there was no uniformity among the confounding factors and the way they were collected (Table.1). This can introduce bias to our results since factors such as age, parity and the period of pregnancy and also the socioeconomic status of women could confound the associations between OHRQoL and oral conditions.

According to the results of this systematic review, there is no conditioned-Specific instrument for measuring oral health related quality of life in pregnant women. In the absence of validated instruments specific to pregnant and postpartum women, all the researchers evaluating OHRQoL in pregnant women use generic instruments. Generic OHRQoL instruments are broad measurement scales, such as OHIP-14 and OIDP that measure OHRQoL in general population. While such questionnaires may be reliable, they may not be appropriate to evaluate OHRQoL in special populations, like pregnant women. They may not be sensitive enough to detect small but important impacts of oral health status during pregnancy. It is obvious that the oral health status of women undergoes some important changes during pregnancy which is the result of physiological and behavioral alteration during the gestation period. Consequently, developing an instrument for measuring OHRQoL specifically in pregnant women might be beneficial and useful for future investigations.

\section{Conclusion}

The main conclusion of this review is that the presence of signs and symptoms of dental and gingival disease negatively affects the self-perception of OHRQoL in pregnant women. The most affected domains of OHRQoL in pregnant women were related to mental and psychological discomfort followed by physical and functional problems. Given that the available evidence is limited to cross-sectional designs, longitudinal studies are needed to investigate the impacts of oral health status during pregnancy and improve the existing evidence on the importance of other parameters such as age, parity, period of pregnancy and socioeconomic status.

\section{Abbreviations}

OHRQoL: oral-health-related quality of life PPD: Periodontal Pocket Depth

CAL: Clinical Attachment Loss DMFT: Decayed, Missing and Filled teeth index 
PI: Plaque Index BOP: Bleeding on probing

CPI: Community Periodontal Index GI: Gingival Index VPI: Visible plaque index NLT: Number of lost teeth OHIP: Oral Health Impact profile OIDP: Oral Impact on Daily Performances

\section{Declarations}

\section{Ethics approval and consent to participate:}

The study was approved by the local Research and Ethics Committee from the Isfahan University of Medical Sciences (IR.MUI.REC.1396.3. 722). Informed consent was obtained from all individual participants included in the study.

\section{Consent for publication:}

Not applicable.

\section{Availability of data and materials:}

The datasets analyzed during the current study are available based on the request.

\section{Competing interests:}

The authors declare that they have no conflict of interest.

\section{Funding:}

Isfahan University of Medical Sciences and Clinician-Scientist Program office (Project ID: 396722\#)

\section{Authors' contributions:}

OF: contributed to the conception and design of the study, conducted to data analysis interpretation and drafted the manuscript. ZSM: contributed to interpreting the results and in writing the manuscript. AK: contributed to interpreting the results and in writing the manuscript. All authors read and approved the final manuscript.

\section{Availability of data and materials}

The dataset supporting the conclusions of this article available and will be presented based on request.

\section{Acknowledgement:}

Not applicable.

\section{References}


1. Hemalatha V, Manigandan T, Sarumathi T, Aarthi Nisha V, Amudhan A. Dental considerations in pregnancy-a critical review on the oral care. Journal of clinical and diagnostic research: JCDR. 2013;7(5):948.

2. Silk H, Douglass AB, Douglass JM, Silk L. Oral health during pregnancy. American family physician. 2008;77(8).

3. Yonkers KA, Wisner KL, Stewart DE, Oberlander TF, Dell DL, Stotland N, et al. The management of depression during pregnancy: a report from the American Psychiatric Association and the American College of Obstetricians and Gynecologists. General hospital psychiatry. 2009;31(5):403-13.

4. Gaffield ML, GILBERT BJC, MALVITZ DM, ROMAGUERA R. Oral health during pregnancy: an analysis of information collected by the pregnancy risk assessment monitoring system. The Journal of the American Dental Association. 2001;132(7):1009-16.

5. Chaveli López B, Sarrión Pérez MG, Jiménez Soriano Y. Dental considerations in pregnancy and menopause. 2011.

6. Bett JVS, Batistella EÂ, Melo G, de Andrade Munhoz E, Silva CAB, Guerra ENS, et al. Prevalence of oral mucosal disorders during pregnancy: A systematic review and meta-analysis. Journal of Oral Pathology \& Medicine. 2019.

7. Ramos-e-Silva M, Martins NR, Kroumpouzos G. Oral and vulvovaginal changes in pregnancy. Clinics in dermatology. 2016;34(3):353-8.

8. Wu M, Chen S-W, Jiang S-Y. Relationship between gingival inflammation and pregnancy. Mediators of inflammation. 2015;2015.

9. Nazir MA. Prevalence of periodontal disease, its association with systemic diseases and prevention. International journal of health sciences. 2017;11(2):72.

10. Azofeifa A, Yeung LF, Alverson C, Beltrán-Aguilar E. Dental caries and periodontal disease among US pregnant women and nonpregnant women of reproductive age, National Health and Nutrition Examination Survey, 1999-2004. Journal of public health dentistry. 2016;76(4):320-9.

11. Rangel-Rincón LJ, Vivares-Builes AM, Botero JE, Agudelo-Suarez AA. An Umbrella Review Exploring the Effect of Periodontal Treatment in Pregnant Women on the Frequency of Adverse Obstetric Outcomes. Journal of Evidence Based Dental Practice. 2018;18(3):218-39.

12. Rocha JS, Arima LY, Werneck RI, Moysés SJ, Baldani MH. Determinants of dental care attendance during pregnancy: A systematic review. Caries research. 2018;52(1-2):139-52.

13. Karimi M, Brazier J. Health, health-related quality of life, and quality of life: what is the difference? Pharmacoeconomics. 2016;34(7):645-9. 
14. Guyatt GH, Feeny DH, Patrick DL. Measuring health-related quality of life. Annals of internal medicine. 1993;118(8):622-9.

15. Boggess KA, Edelstein BL. Oral health in women during preconception and pregnancy: implications for birth outcomes and infant oral health. Maternal and child health journal. 2006;10(1):169-74.

16. Moher D, Shamseer L, Clarke M, Ghersi D, Liberati A, Petticrew M, et al. Preferred reporting items for systematic review and meta-analysis protocols (PRISMA-P) 2015 statement. Systematic reviews. 2015;4(1):1.

17. Wells G. The Newcastle-Ottawa Scale (NOS) for assessing the quality of non randomised studies in meta-analyses. http://www ohri ca/programs/clinical_epidemiology/oxford asp. 2001.

18. Ferreira M, Dias-Pereira A, Branco-de-Almeida L, Martins C, Paiva S. Impact of periodontal disease on quality of life: a systematic review. Journal of periodontal research. 2017;52(4):651-65.

19. Geevarghese A, Baskaradoss JK, Sarma PS. Oral health-related quality of life and periodontal status of pregnant women. Maternal and child health journal. 2017;21(8):1634-42.

20. Moimaz S, Rocha NB, Garbin A, Garbin C, Saliba O. Influence of oral health on quality of life in pregnant women. Acta odontologica latinoamericana: AOL. 2016;29(2):186-93.

21. Lu H-X, Xu W, Wong MCM, Wei T-Y, Feng X-P. Impact of periodontal conditions on the quality of life of pregnant women: a cross-sectional study. Health and quality of life outcomes. 2015;13(1):67.

22. Lamarca GA, Leal MdC, Leao AT, Sheiham A, Vettore MV. The different roles of neighbourhood and individual social capital on oral health-related quality of life during pregnancy and postpartum: a multilevel analysis. Community dentistry and oral epidemiology. 2014;42(2):139-50.

23. Cornejo C, Rossi G, Rama A, Gomez-Gutierrez N, Alvaredo G, Squassi A, et al. Oral health status and oral health-related quality of life in pregnant women from socially deprived populations. Acta Odontológica Latinoamericana. 2013;26(2):68-74.

24. Wandera MN, Engebretsen IM, Rwenyonyi CM, Tumwine J, Åstrøm AN. Periodontal status, tooth loss and self-reported periodontal problems effects on oral impacts on daily performances, OIDP, in pregnant women in Uganda: a cross-sectional study. Health and quality of life outcomes. 2009;7(1):89.

25. Acharya S, Bhat PV. Oral-health-related quality of life during pregnancy. Journal of public health dentistry. 2009;69(2):74-7.

26. de Oliveira BH, Nadanovsky P. The impact of oral pain on quality of life during pregnancy in lowincome Brazilian women. Journal of orofacial pain. 2006;20(4). 
27. de Oliveira BH, Nadanovsky P. Psychometric properties of the Brazilian version of the Oral Health Impact Profile-short form. Community dentistry and oral epidemiology. 2005;33(4):307-14.

28. Slade GD. Assessment of oral health related quality of life. Oral health-related quality of life. 2002.

29. Slade GD, Spencer AJ. Development and evaluation of the oral health impact profile. Community dental health. 1994;11(1):3-11.

30. Adulyanon S, Sheiham A. Oral impacts on daily performances. Measuring oral health and quality of life. 1997;151:160.

31. Adulyanon S. An integrated socio-dental approach to dental treatment need estimation: UCL (University College London); 1996.

32. Haag D, Peres K, Balasubramanian M, Brennan D. Oral conditions and health-related quality of life: a systematic review. Journal of dental research. 2017;96(8):864-74.

33. Adulyanon S, Vourapukjaru J, Sheiham A. Oral impacts affecting daily performance in a low dental disease Thai population. Community dentistry and oral epidemiology. 1996;24(6):385-9.

\section{Tables}

Due to technical limitations, tables 1 and 2 only available as a download in the supplemental files section.

\section{Figures}




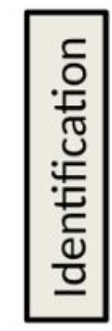

\section{Articles identified through search of databases} and bibliographic references of selected studies

$(\mathrm{N}=364)$
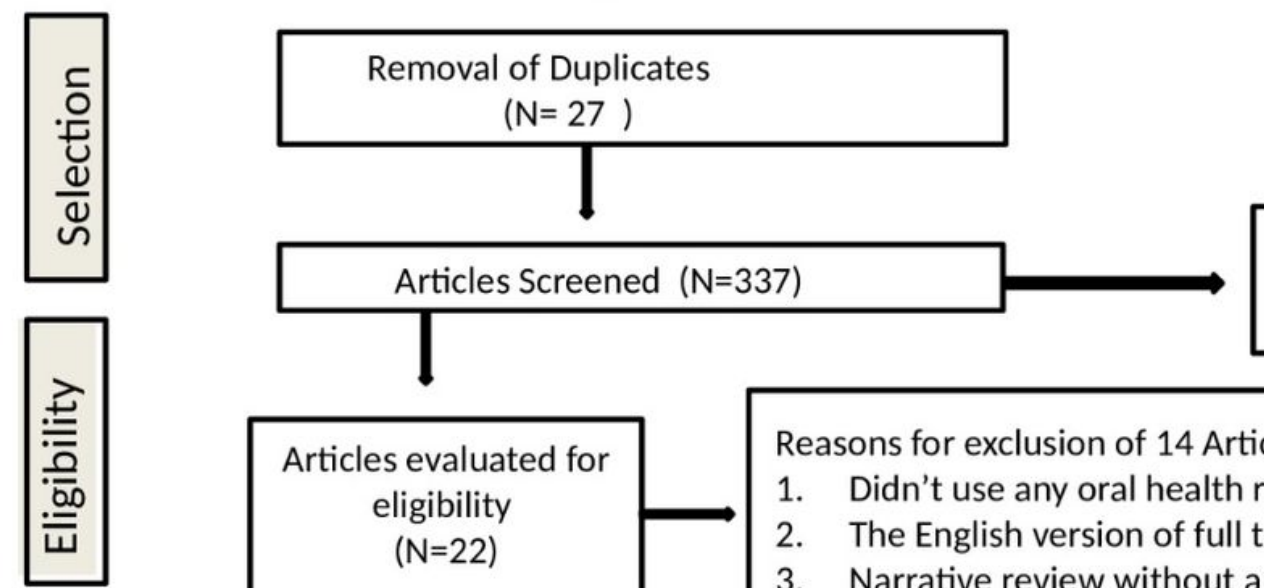

Articles excluded

$(\mathrm{N}=315)$

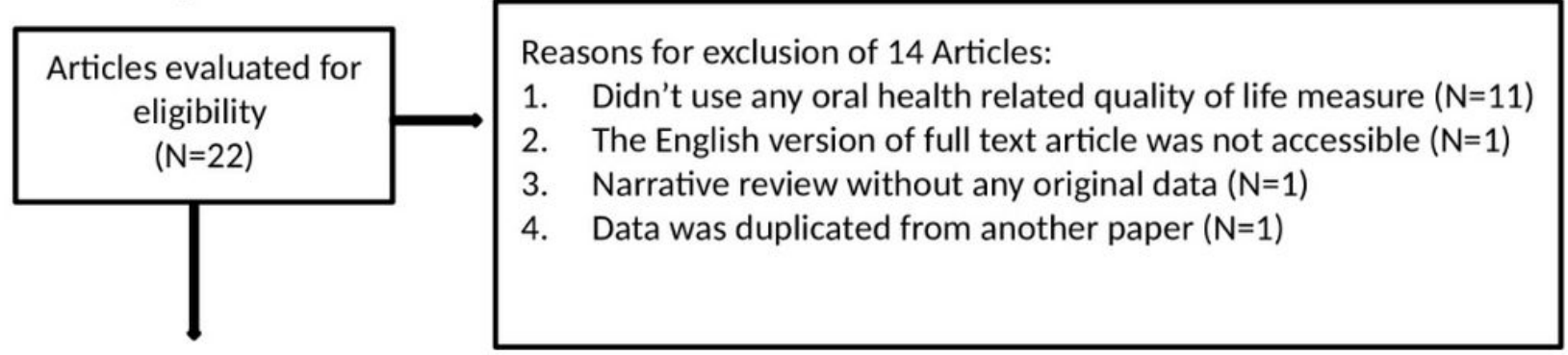

Articles included in qualitative synthesis $(\mathrm{N}=8)$

\section{Figure 1}

Study selection process: PRISMA flowchart of four phases of review showing number of studies identified, selected, eligible and included.

\section{Supplementary Files}

This is a list of supplementary files associated with this preprint. Click to download.

- MainAppendix.pdf

- Table1.jpg

- Table2.jpg 